\title{
UN NUEVO MANUSCRITO DEL COMENTARIO DE G. BARZIZZA A LAS EPÍSTOLAS DE SÉNECA
}

\begin{abstract}
The manuscript 12 in Salamanca University Library, unknown until now, contains a commentary by Gasparinus Barzizza on the Epistulae Morales of Seneca. It is a manuscript of the xvth century and can aid to clarify several aspects of the textual transmission.
\end{abstract}

A propósito de una proyectada edición de las epístolas de Séneca, en el proceso de búsqueda de manuscritos en bibliotecas españolas no localizados por Reynolds', encontré en el catálogo de manuscritos de L. Rubio una referencia a un comentario de las Epistolas: el ms.12 de la Biblioteca Universitaria de Salamanca ${ }^{2}$.

En el inventario de $1855^{3}$ de esta misma Biblioteca, el manuscrito viene registrado como Quaedam epistolae, título que en el propio manuscrito aparece en la cara interior de la tapa y en el corte frontal.

Se trata de un manuscrito del siglo xv, $280 \times 210 \mathrm{~mm}$., en papel, con encuadernación original, escrito a dos columnas. Falta la hoja de guarda; 124 folios, numeración moderna; numeración antigua en el borde inferior derecho, siguiendo el sistema de letra y número. Se distinguen dos partes en el manuscrito: ff. 1-103 y 104-122. La primera parte consta de dos cuadernillos de 12 folios, y seis cuadernillos de 14 folios: c, d, e/g, h, i. La numeración del primero sólo llega hasta a5 debido a que no está incluida en la numeración la hoja de guarda, y el último sólo alcanza a i5, probablemente por omisión. El primer cuadernillo ocupa en la encuadernación los doce primeros folios y los doce fo-

L. D. Reynolds, The medieval tradition of Seneca's letters, Oxford 1965.

2 L. Rubio, Catálogo de los manuscritos españoles de autores clásicos, Madrid 1985.

Manuscritos de la Biblioteca Salmantina. Índice de los libros manuscritos que se conservan en la Biblioteca de la Universidad de Salamanca formado en 1855 por V. de la Fuente y J. Urbina. 
lios correspondientes al quinto lugar $(=f)$. Los seis cuadernillos de 14, en disposición 3 y 3 encuadran a $\mathrm{f}$. Cada una de las mitades del cuadernillo va numerada sólo en su primera mitad.

La segunda parte lleva numeración independiente. Comienza en el folio 104 (=a 1) y sólo llega hasta 109 (a6), es decir un cuadernillo de 12 folios. De hecho el manuscrito tiene 124 folios, de los cuales sólo 122 están escritos.

A esta diferenciación en dos partes se suma el cambio de letra y tinta en el folio 104 r. Asimismo desaparecen las iniciales de cada epístola en capitales ornadas en rojo y azul, y las separaciones de parágrafos en los mismos colores. Otro dato que confirma la existencia de dos partes es la ruptura en la progresión creciente del número de líneas. El manuscrito comienza teniendo 43 líneas y va subiendo hasta alcanzar en torno al f. 90 un promedio de unas 90 líneas, para bajar de nuevo en el f. 104 a las 40 lineas.

Se trata de un comentario a las Epístolas de Séneca, precedido de una introducción-presentación, a modo de accessus amplísimo, seguida de una Vita Senecae. El cuerpo del manuscrito lo forma un comentario a las 65 primeras epístolas de Séneca. Las 57 primeras ocupan la llamada primera parte del manuscrito, y hasta la 65 cubren los restantes folios. Hay que advertir que entre la epístola 58 y la 59 se inserta, numerada como 59, una epistola inexistente en el corpus senecano, lo cual hace que la numeración se corra un número y la epístola final, 65 , se dé como 66.

Aunque el autor del comentario advierte en el proemio: Non dico liber primus quamuis a nonnullis in xxii libros diuiditur, quoniam illa diuisio omni ratione prorsus caret, esto no es obstáculo para que encontremos al comienzo de la epístola 12: Incipit liber secundus epistolarum Senece ad Lucilium, y a comienzos de la epistola 20: Hec est prima epistola libri tertii...

En la numeración de los fondos de la Biblioteca hecha en 1610 por Miguel de Velasco (BUS, ms. 25), aparece en el f. 69 r el siguiente registro: "Caxon 40 Gasparinus in epistola (sic) Senecae fo. de mano." $\mathrm{Si}$ alguna duda pudiera ofrecer su atribución a Gasparino de Barzizza ésta fue disipada al confrontar el contenido de la introducción y Vita con la transcripción que de ambos, a partir de otros manuscritos, hace L. A. Panizza en un artículo del año $1977^{4}$. Esta transcripción está basada en

4 L. A. Panizza, "Gasparino Barzizza's commentaries on Seneca's letters", Traditio 33, 1977, pp. 297-358; se encuentra mencionado este comentario, en la versión del manuscrito de Cremona 128, en N. W. Gilbert, «A letter of Giovanni Dondi dall'Orologio to Fra' Guglielmo Centueri: a fourteenth-century episode in the quar- 
el ms. Nápoles V. D. 20, por lo que se refiere a proemio e introducción, aunque añade como muy similares el Vind. lat. 51, procedente de Nápoles y actualmente de nuevo allí, y Venecia, Fondo Correr 1437, todos ellos del siglo xv. Por lo que se refiere al comentario de las epístolas, Panizza da el número de 110 , aunque no he podido deducir si es resultado de una consulta directa o bien es una traslación de las observaciones de catálogos 5 .

La misma autora nos ofrece manuscritos con distintas versiones de este mismo comentario: Cremona, R. Bibl. Gov. 128, Brit. Libr. Roy. lat. 15. C. IX, por un lado, y Urb. lat. 218 (olim 1026) ${ }^{6}$, todos ellos también del siglo $\mathrm{xv}$. El primero contiene comentario a las cuarenta primeras epistolas, y a la 110 , aunque en versión más breve que la ofrecida por Nap. V. D. 20; del manuscrito de la British Library nada nos dice Panizza, salvo su afinidad con el de Cremona, pero el número de folios hace sospechar que el número de epístolas comentadas es mucho menor. En cuanto al manuscrito de la Vaticana nos lo caracteriza como distinto en desarrollo, tanto con respecto al modelo representado por Nap. V. D. 20, como al de Cremona.

Como hemos adelantado más arriba, la coincidencia de nuestro manuscrito con la transcripción que de proemio y vida de Séneca hace $\mathrm{Pa}$ nizza siguiendo a Nap. V. D. 20 y Cremona 128 respectivamente, es casi total ?

Tal como dice Panizza, el manuscrito de Cremona se diferencia del de Nápoles y, por consiguiente, del salmantino, en ofrecer una versión completamente distinta, muy breve, del proemio, un comentario a la primera epistola restringido a la primera parte, y una versión de los comentarios a las distintas cartas reducido casi siempre en su parte final. Basándose en las declaraciones de Barzizza en el proemio extenso, $\mathbf{P a -}$ nizza concluye la existencia de dos redacciones del comentario y las hace coincidir con las versiones transmitidas por el códice de Cremona y por el de Nápoles.

rel of the ancients and the moderns", Viator 8, 1977, pp. 299-346. En general suele hablarse de escasez de comentarios a esta obra de Séneca, véase B. J. Hijmans, Jr., "Two Seneca mss. and a commentary", Mnemosyne 21, 1968, pp. 240-253.

s O.c., p. 310, n. $^{\circ} 55$.

- Mencionado por Nicolás Antonio, Bibliotheca Vetus, Roma 1696, vol. I, libro I, cap. 8 (f. 153).

7 No insisto sobre la Vita porque es objeto de especial atención por parte de L. A. Panizza. Sin embargo, entra dentro de nuestros propósitos editar este comentario, momento en que habrá que tratar de todos y cada uno de los puntos con él relacionados. Un escueto comentario sobre la epistola 1 en Barzizza, en L. A. Panizza, "Textual interpretation in Italy, 1350-1450: Seneca's letter I to Lucilius", JWI 46, 1983, pp. 40-62. 
Los pasajes sobre los que se fundamenta están en los parágrafos por ella numerados como 9,10 y 11; doy de ellos un resumen en castellano: Barzizza habla de una época de su vida (imperfecto), en que la lectura de Séneca y de otros autores latinos y griegos le llevó a apreciar su valor como filósofo moral, y eso le hace pensar que cuando tenga tiempo libre se dedicará a su lectura.

Esta decisión de esperar una época de ocio se ve frustrada por la amicorum uoluntas, que le obliga a exponer públicamente las epístolas. Todavia más recientemente (nuper) los amigos le han obligado a algo todavía más dificil, a editar unos comentarios; en esto Barzizza ha visto la oportunidad de defenderse de los posibles ataques a su obra anterior, que ya había sido editada. Para ello piensa en reformar el proemio (uelut caput a me atque initium rescribendum), ya que los amigos le exigen cumpla algo prometido en tiempos: iterum in publico legere (exponere $S)$.

Decide en este comentario completar la primera epístola (Reliquam autem partem... tunc praetermisi quamuis nunc admonitus sim locum quem inexpletum reliqueram perficere), añadiendo un comentario a la segunda parte de la epístola.

Como se ve, parece claro que Barzizza ha dedicado su atención a las epístolas de Séneca en dos ocasiones; que las dos versiones sean las conservadas por los manuscritos de Cremona $(C)$ y Nápoles $(N)$ / Salamanca $(S)$ constituiría un primer punto de discusión. Si bien es cierto que en el de Cremona tenemos un accessus en lugar del amplio proemio de $N S$; si bien la primera epístola de $C$ está incompleta y el resto de las epistolas es de menor extensión, debemos aclarar que en el caso de la epistola 1 la ruptura se produce en medio de una frase (confirmat Lucilium debere se sibi uindicare et prouidentem esse circa distributionem sui temporis. Confirmat etiam), el resto del folio queda en blanco y las restantes epistolas ven menguado su volumen precisamente en su segunda parte. El menor volumen en ocasiones parece deberse más bien a un recorte que a otra cosa. Por ejemplo, en la epístola tres, la versión larga, después de unas apreciaciones sobre quiénes deben ser considerados amigos, apreciaciones apoyadas en la opinión de Aristóteles, introduce una referencia marginal a Teofrasto, como discípulo del anterior. Pues bien, en $C$ la mención de Teofrasto aparece sin ninguna apoyatura que la justifique, privada de cualquier sentido dentro del contexto. A este detalle podríamos añadir la ausencia en $C$ de la forma final de conclusión presente siempre en $N S$. Cuestiones de este tipo hacen que mi opinión sobre la posibilidad de que estas dos series de manuscritos representen la hipotética doble versión quede en suspenso. 
Cuestión un tanto distinta es la de la datación. Panizza atribuye la primera versión al año 1411 , partiendo de dos cartas fechadas por Sabbadini en 1410, en las que Barzizza habla de que se vio obligado a interrumpir la redacción de un comentario sobre el de officiis ciceroniano para exponer en público sententias epistolarum Senecae, aunque en esos momentos, una vez terminado su trabajo sobre Séneca, ha vuelto a tomar entre sus manos el de officiis. La inserción de una precisión al nombre de Francisco Zabarella, incluido como garante de una opinión en la primera epístola, modo cardinalem florentinum, reafirma la opinión de Panizza sobre la fecha, ya que Zabarella fue nombrado cardenal de Florencia en 1411 y la autora del artículo interpreta modo como "recientemente nombrado" ${ }^{8}$.

Según esta reconstrucción tendríamos un curso sobre Séneca en 1408, del que da testimonio Gasparino en una epístola, otro nuevo en 1411 (al que según Panizza se correspondería la versión $C$ ) y un nuevo curso o comentario posterior, el conservado por $N S$. Nada puedo aportar a este planteamiento ante la falta de nuevos datos; lo único que parece evidente es que el proemio amplio, correspondiente a un comentario posterior, como hemos visto, insiste sobre lo ajeno que resulta a sus ocupaciones el emprender un comentario sobre Séneca (et quoniam in rem perdifficilem ac meis occupationibus magis incongruam), lo cual parece apuntar a un momento en que su quehacer está volcado en otro autor al que dedica su curso simultáneamente a la redacción del comentario (... si illud meminerint me pauca sedentem domi composuisse cumque iam horas tres legissem... $)^{9}$.

En cuanto al tipo de comentario resulta del máximo interés. Como hemos dicho, va precedido de un proemio con dos partes bien diferenciadas. Dentro de la primera parte, a su vez, adquiere contornos muy definidos la presentación histórica que justifica objetivamente el dedicar un comentario a las Epistolas de Séneca, seguida de una justificación en tono muy personal.

La segunda parte, destinada a hablarnos del tipo de comentario que corresponde a la obra escogida, procede por sucesivas subdivisiones al encuadramiento de la misma. Resulta sorprendente, dentro de este es-

${ }^{8}$ Otra interpretación del pasaje nos aproximaria a la reciente muerte de Zabarella y nos llevaria al año 1417 . El hecho de que el pasaje esté incluido en la versión que Panizza considera identificable con la primera - es decir, la transmitida por el manuscrito de Cremona - es lo que da mayores visos de verosimilitud a su interpretación. Ahora bien, eso supondria un descuido al no eliminar la referencia en la versión posterior. En todo caso, cabe la otra interpretación: "hasta hace poco cardenal de Florencia».

$9 \$ 12$. 
quema escolástico, encontrarse con una interesante digresión sobre la república de Venecia, hecho que puede simbolizar la flexibilidad en el manejo de esquemas previos ${ }^{10}$.

El comentario, pues, está basado en una escolástica distribución del material que va precedida de tres puntos: relación del contenido de la epístola con la epístola anterior o con la supuesta epístola de Lucilio que ha provocado la contestación de Séneca; tema tratado en la carta; intención de Séneca ".

A continuación la epístola se divide en partes y particulae ${ }^{12}$; siempre queda la posibilidad del excursus que permite reconstruir el mundo de conocimientos y de intereses al que se dirige el comentario.

Resulta a primera vista extraño encontrarnos en Salamanca con un códice del xv que contiene una obra de un humanista italiano. Nada se sabe de la procedencia del manuscrito antes del 1610, fecha del catálogo de Miguel de Velasco. Sin embargo, sabemos de la presencia en Salamanca, en la segunda mitad del siglo $\mathrm{xv}$ de numerosos maestros italianos $^{13}$, conocemos la presencia en España de Guiniforte Barzizza, hijo de Gasparino, y del envío a Alfonso de Nápoles por su parte de copias de Sententiae in epist. Senece de su padre ${ }^{14}$; sabemos de los frecuentes

10 Por su posible interés respecto a la fecha del proemio transcribo este pasaje. Como una de las partes que abarca la philosophia moralis menciona la Política, que a su vez se subdivide en regalis, aristocratica et democratica (om. S). Llegado el turno a la Aristocrática se expresa así: Aristocratia est multorum uirtuosorum principatus ubi legum salus et maiorum instituta seuerissime custodiuntur. In quo principatu (-us S) diutius floruit serenissima Ducalis dominatio Venetorum quam alia politia de qua in antiquis annalibus usquam legerem (usque legatur S). Licet enim Spartani glorientur annis ferme septingentis nulla ex parte mutatis legibus institutisue maiorum regnasse, tamen neque optimo genere principandi neque diuturnitate regnandi ego Spartanos umquam Venetis comparaui, cum hi annis fere mille eum principatum quem ab urbe condita semel accepissent, nedum acceptum conseruauerint (non reliquerint $S$ ), sed pulcherrimis insuper maximarum uirtutum ornamentis non sine summa omnium gentium admiratione auxerint. Que enim ciuitas ita bene morata ullo tempore fuit...? La fecha del 421 para la presunta fundación de Venecia, aceptada desde finales del s. XII no resuelve demasiado, ya que el margen concedido por fere podría convenir tanto a 1411 como a 1417 ó 1418; cf. E. Franceschini, «La cronachetta di Maestro Iacopo Dondi", Scritti di Filologia Latina Medievale, vol. I, Padua 1976, pp. 230-246, artículo que reproduce uno publicado en 1940.

"Puede servir como muestra el inicio de la epistola 1: ITA FAC MI LVCILI Hec est prima epistola Senece ad Lucilium ad cuius introductionem tria sunt premittenda. primum est qualiter hec epistola continuatur ad epistolam Lucili. Secundum est que sit materia huius presentis epistola. tertium autem qualis autoris finalis intentio.

${ }_{12}$ De la misma epistola 1: Diuiditur autem hec epistola in duas partes principales... Prima pars habet tres particulas... in prima... in secunda... in tertia. Pasa a continuación a exponer con amplitud la primera parte con sus particulae, etc.

${ }_{13} \mathrm{Cf}$. P. Verrua, Precettori italiani in Spagna durante il regno di Ferdinando il Cattolico, Adria 1907.

14 M. E. Cosenza, Biographical and bibliographical Dictionary of the Italian Humanists, I, Boston 1962. 
viajes a España de Angelo Decembrio, alumno de Gasparino en Padua ${ }^{15}$ y que «en su última expedición, en 1464, llevaba muchos códices y preciosas transcripciones» ${ }^{16}$; todo ello sin olvidar, naturalmente, la presencia de Nebrija en Bolonia durante unos años. Es decir, no fueron pocas las oportunidades de que a Salamanca llegara relativamente pronto una obra de Gasparino Barzizza.

CARMEn CODOÑer

is W. H. Woodward, Vittorino da Feltre and other humanist educators, Cambridge 1925, p. 10 ss.; L. A. Panizza, o.c., p. 301.

16 A. Farinelli, Viajes por España y Portugal, Roma 1942, I, p. 122. 